\title{
Biomedical applications of electrically conductive polymeric systems
}

\author{
Joseph Jagur-Grodzinski* \\ WWeizmann Institute of Science, Dept. Materials \& Interfaces, Rehovot 76100 Israel; \\ e-mail: Joseph.jagur@weizmann.ac.il
}

(Received: 20 February, 2012; published: 23 July, 2012)

\begin{abstract}
Properties of the electrically conductive polymers (ECPs) enable introduction of several novel applications in various fields, among others, as biomedical materials. Their use as scaffolds for tissue engineering and recovery of damaged neural tissues is especially advantageous. They may also be used for the electrically induced drug release and delivery, and as very sensitive biosensors for clinical applications. Another use is the detection and precision of the biologically important chemical materials. They have been shown to modulate and accelerate activities of the nerve, skeletal, muscle, and bone cells. Cell growth, migration and adhesion, may be stimulated by applying electric potential. Synthesis of DNA, secretion of proteins, and transformations of stem cells may be enhanced. Clinical analysis using ECP-based biosensors make possible the precise determination of the exact composition of individual DNA molecules, and thus enable early detection and treatment of genetically related diseases. Electrochemical (EC) approach of ECP-sensors enables precise determination of concentrations of several biologically important chemicals.

Keywords: Tissue engineering, regeneration, polypyrrole, polyaniline, drug delivery
\end{abstract}

\section{Introduction}

The serendipitous rediscovery of conductive polymers in 1963 at the plastic research laboratory of BASF was a result of attempts to prepare aromatic polymeric compounds [1]. Almost at the same time the electronic properties of polypyrrole (PPy) were reported and investigated by Bolto et al. [2] Electric properties of several other conjugated polymers were also investigated by CSIRO researchers [3-4], and by other research groups [5]. Shirakawa et al.[6-10] who investigated properties and synthetic routes for preparation of polyacetylene and its derivatives, noted that their electric conductivity may be increased by orders of magnitude by doping them with lodine or other halogens ( $p$-doping). The electric conductivity of the conjugated chains and effect of "doping" (exposing polymer to electron-withdrawing or electronwithdrawing or donating groups) on their conductivity (p-doping \& n-doping) was later investigated by other research groups [11-12]. The mechanism of electric conductivity of polyacetylene was elucidated by Shirakawa, Hedger \& MacDiarmid $[10,13]$. They concluded that transfer of electrons in polyacetylene is associated with movement of "solitons" generated by bond alteration in domain walls due to formation of kinks, as a result of cis-trans transformations of the cis-isomer or imperfections in the trans-isomer formed during its polymerization. Paramagnetic solitons become positively charged upon $\mathrm{p}$-doping and are converted into spin-less polarons, which travel along the polymeric chain. These conclusions were confirmed by the results of ESR and IR experiments and by the analysis of magnetic, electrical and optical 
properties of the lightly doped polyacetylene were studied by Heeger and by MacDiarmid in cooperation with other investigators [14-16]. A very large increase (by six orders of magnitude) in the conductivity of conjugated polythiophenes was also observed by Pozdorow et al.[17] when polythiophene matrix was doped by vapors of halogenated alkyl silanes, as result of proton doping by free silanol groups in the hydrolyzed trihalosilanes of a partly polymerized siloxane network.

It was initially believed that the presence of conjugated double bonds (two or more double bonds separated by single bonds) is a condition ("sine qua none") for the electronic conductivity of these materials [18]. However, it was observed [19] that non-conjugated double bonds may also behave in a similar fashion, when electrondonating groups are attached to them [19-21]: a) Electric conductivity of lodinedoped poly(isoprene) is in the $10^{-2}-10^{-1} \mathrm{~S} / \mathrm{cm}$ range, b) It is even higher for transpoly[1,3-butadiene] doped with lodine, as well as for the doped biphenyls, substituted with ketones to which long aliphatic chains have been attached. It should be pointed out that electrical conductivity of polymeric systems with conjugated carbon double bonds, such as polyacetylene, may not be very stable in the presence of atmospheric oxygen and/or moisture [22, 23]. When the doped polymers are not stabilized by interaction with counter-ions, their "doping" may be the source of chemical instability. Inclusion of nitrogen or sulphur atoms in the conjugation system has stabilizing effect on the systems. Examples of polymers containing nitrogen or sulphur atoms are shown in Figures 1 to 3.

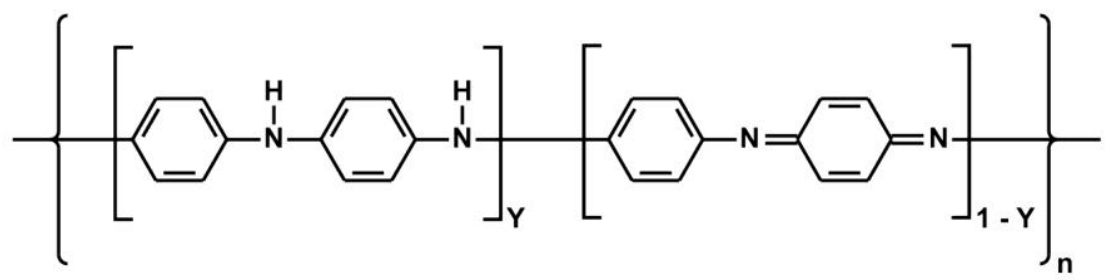

Fig. 1. The general structure of polyaniline (PANI).

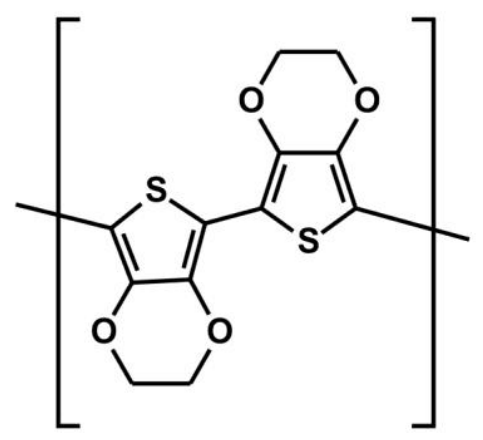

Fig. 2. The structure of poly(3,4-ethylendioxythiophene) (PEDOT).

Therefore, the electrical polypyrrole (PPy), polythiophene (PT), poly3,4ethylendioxythiophene) (PEDOT), etc. has been extensively investigated. Scheme illustrating structure of the dopped polypyrrole is shown in Figure 3. 


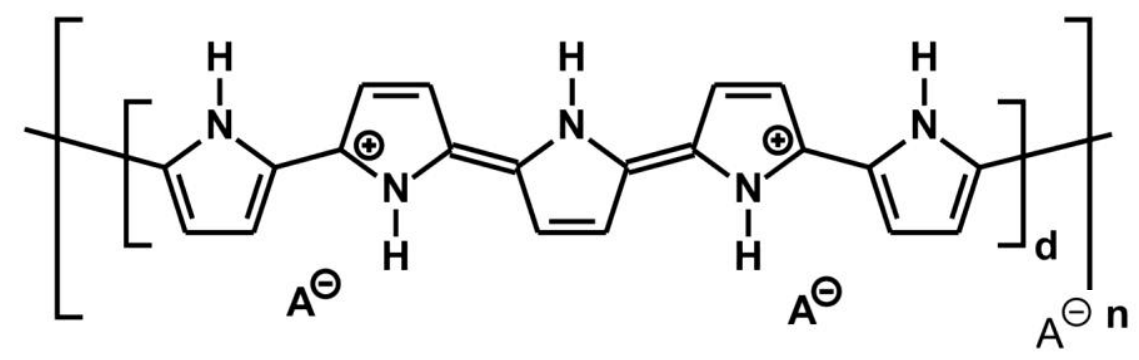

Fig. 3. Structure of p-doped polypyrrole (PPy); counterion of dopant, d degree of doping, $n$ degree of polymerization.

After the discovery of the electrically conductive polymers (ECP's), it seemed initially that practical applications of these new materials may be limited by their brittleness and by problems. Moreover, the usefulness of these novel materials for various novel applications, some not yet developed at the time of their discovery, was not obvious at that time. In order to overcome problems of brittleness and processibility of conductive polymers, while at the same time protecting them from atmospheric oxygen, ECP's were blended with various non-conductive polymers. Electric conductance of such blends is attained when concentration of the conductive material exceeds their threshold points. The electric conductivity of the ECP's may be further ameliorated by insertion of electrically conductive particles into a polymeric matrix. Carbon nanotubes, as well as particles of carbon black, graphite and metals, have been used for such purposes. Many applications based on the electroactive behaviour of ECP's are known today. One of the large scale applications of ECP's is based on the protective anticorrosion behaviour of metals coated by them [24-28]. These applications involve anodic anti-corrosion protection. Supply of electrons to the coated metal lead to novel formation of a very thin layer of oxide on their surface, as well as to conversion of protons into hydrogen's [28]. Biocompatible ECP's have been found to be of particular importance for biomedical applications, such as tissue engineering [ 9] regenerative medicine, and biosensors. Formation of the electrically conductive transparent coatings has also been used for anti-static's (ESD), EMI/RFI shielding, lithographic resists, for photovoltaics, rechargeable batteries, solar cells, actuators, transducers, electro-chromic displays, textiles (particularly attractive for military applications), gas and liquid separators, biological and chemical sensors, microelectronics, electromechanical devices, electroluminescence (light emitting diodes (LEDs), electrodes for super-capacitors etcetera. List of applications of transparent conductive polymer coatings was cited and shortly described by Kulkami [30]. In Transparent Conductive Coatings (chapter 38 of ref.28).

Responsiveness of some tissues to electric stimuli makes the biocompatible CP's particularly attractive for several biomedical applications. Availability of such materials may provide solutions to many problems in neural biology/medicine [31]. Such materials have been shown to modulate activities of nerve, cardiac, skeletal muscle, and bone cells. They stimulate cell growth, migration, and adhesion, enhance DNA synthesis and protein secretion. They might also be useful for the time predetermined drug release and delivery. Their reversible doping, as well as possibility of adaptation of their electrical, chemical, and physical properties according to the requirements of specific applications, may be cited as their big advantages [ 2]. It must also be pointed out that co-polymerization with nonelectrically conductive polymer or addition of relatively small amounts of CP's to such 
polymeric material may impart some of its properties to their blends, composites, or copolymers. Wan et al. suggested [3] that addition of 3-6 \% of PPy to porous chitosan produces conductive porous materials for use as scaffolds for tissue engineering. The biological application of polyaniline and its blends was mentioned by Bhadra et al. [34] in their review on preparation, processing, and applications of polyaniline. Chan \& Mooney [35] discuss electrically conductive materials among new materials for tissue engineering which enable achieving greater control over the biological response, and are of particular interest for neural-tissue engineering, and for responsive prosthetics. Barriere [36] et al. \& Straley [37] et al. pointed out the usefulness of ECPs for regeneration of skeletal tissues, and treatment of skeletal tissue injuries. Recently published papers describing stimulation, activation, and immobilization of cells and some components of their extracellular matrix (ECM), preparation of scaffolds for tissue engineering (TE) and regeneration, as well as construction of neural electrodes and biosensors are described and briefly discussed in the present review. Changes in dimensions of ECPS due to doping and electrical stimulation indicate that they may act as actuators converting electric into mechanic energy. Several attempts had been made, therefore, to develop efficient actuators using ECPs. Though it has been demonstrated that PEDOT, PPy, and PANI may act as actuators [38, 39], scientific and technological obstacles must still be overcome [39] before, strong, silent, and lightweight artificial muscles, based on ECPs, will be commercially available.

\section{ECPs for tissue engineering and regenerative medicine}

Polypyrrole (PPy) is an ECP widely used for such purposes. Its bio-compatibility with nerve tissue was evaluated in-vitro and in-vivo by Wang [40] et al. Neither direct nor acute or sub acute toxicity or pyretogen were detected when Schwann cells were exposed to the extraction solution of PPy. These cells had better survival and proliferation rates then Schwann cells exposed to saline water. Moreover, there was no difference between dorsal root ganglia, from 1-3 days old Sprague-Dawley rats, cultured either on PPy membranes or on glass as the control group. In the in-vivo experiments PPy coated silicon tubing's were used to bridge the10-mm sciatic nerve gaps in rats. After six months the regeneration of the nerve was slightly better in the PPy coated silicon tubing than in the control. Peppas, Bayer, et al.[41] prepared a water soluble derivative of PANI, which can be easily integrated with bio-materials. They showed that they are not toxic towards the NIH 373 fibroblasts cultured on it, maintained growth habits similar to those observed on controlled surfaces, and retained functionality after cell growth.

PPy and systems based on it, were described and extensively researched by Christine Schmidt and her collaborators, as well as by other research groups. Fonner, Schmidt, \& Ren [42] made a combined computational-experimental study of the structure, charge distribution and backbone flexibility of the undoped PPy and chlorine doped derivative PPyCl. They found that backbone flexibility increases strongly as a result of doping and that $80 \%$ of the charge in the chlorine doped $\mathrm{PPyCl}$ is located on the 3 pyrrole rings closest to the chlorine.

\section{Neuronal stimulation by NGF and/or by electric current}

Shoichet et al. [43] discussed modifications of biomaterials that promote neuron growth and adhesion. Gomez \&, Schmidt [44] investigated the effect of immobilization of nerve growth factor (NGF) (by its attachment to PPy), on its activity 
towards neuron-like PC-12 cells. They used polyallylamine (PAA) (complexed with N4(azidobenzoyloxy) succinimid,) as a photo linker. PPy-link-NGF was placed on neuron-like PC-12 cells. In a parallel experiment NGF was dissolved in a solution adjacent to it. Results of these experiments indicated that PC-12 cells can grow neurites on PPy with NGF immobilized on it, nearly as well as when NGF is dissolved in an adjacent solution. Extensions of neurites in cells exposed to NGF immobilized on PPy and in cells which were exposed to NGF added to the solution adjacent to PPy, were nearly the same after 2 days. However, significant differences in the percentage of cells with extended neurites were observed after 10 days. Some detrimental effect due to immobilization of NGF was probably caused by the inhibition of endocytosis dependant pathways in the immobilized NGF. Lee (JY), Lee (JW) \& Schmidt $[44,45]$ developed copolymers (PPy-co-NSE) of pyrrole (Py) with the Nhydroxyl succinimidyl ester of pyrrole (NSE). They could be immobilized on NGF without significantly hindering their electro-conductivity or reducing the neurotropic activity of NGF. The PC-12 cells cultured on the immobilized PPY-NSE ${ }_{50^{\prime}}$ extended neurites to the same extent as cells cultured on PPy in contact with solution containing free NGF. Moreover, NGF immobilized via the active ester on the PPy$\mathrm{NSE}_{50}$ films was stable for up to 5 days in a phosphate-buffered saline solution. It showed good stability under physiological conditions and its activity was not affected by the application of an electric potential. Other biomolecules can also be immobilized in the same fashion.

\section{Adhesion, proliferation, and stimulation}

Kotwal \& Schmidt [46] demonstrated that nerve regeneration may be enhanced by the application of electric charges. They assumed that this effect may be caused by an increase in the adsorption of fibronectin (FN) (a serum protein), due to changes in the local electric fields of the ECM molecules, induced by the applied electric stimulus. To check this hypothesis they investigated the effect of electrical stimulation on the adsorption of pure FN, and of FN dissolved in serum solution. Results of these studies demonstrated that the amounts of adsorbed FN increased during electric simulation of PPy, inducing increase in neurites outgrowth. Akkouch, Rouabhia, et al.[47] bio-activated PPy by incorporation of fibronectin (FN) and bovine serum albumin (BSA). Adhesion and proliferation of fibroblast cells in contact with PLLA membranes containing the bioactivated PPy particles was significantly modified in comparison to PLLA-PPy which was used as a control group. Attachment and growth of fibroblast cells cultured on membranes containing FN-rich PPy particles increased significantly. On the other hand it decreased in cells cultured in contact with BSA-rich PPy particles. Thompson Richardson, Wallace et al. [48,49] electrically stimulated PPy with simultaneously incorporated neurotropin (NT-3) and brain derived neurotropic factor (BDNF). They found from Cochlear neural implants that, electrical stimulation, in the presence of NT-3 and BDNF, had a dramatic effect on auditory nerve survival and growth. Neurite outgrowth was more than double compared with the effect of the NT3 alone. Huang, Hu, et al.[50] showed, that electrical stimulation of PPy/chitosan blend enhanced the viability of Schwann cells. It also increased levels of NGF, BDNF, and mRNA as well as their expression. Kotov et al.[31] published an extensive overview on the use of nanomaterials for neural tissues. They pointed out that impedance of the microelectrodes for neural investigations may be considerably reduced by coating their surface with PPy or PEDOT films. In comparison to bare gold electrodes, the impedance of such microelectrodes may be reduced (at $1 \mathrm{~Hz}$ frequency) by about two orders of magnitude and charge transfer capacity increased 
by three orders of magnitude. Guo, Wei et al.[51] prepared a novel electroactive precursor N-(4-aminophenyl)-N-(4'-(3-triefhoxysilyl-propyl-ureido)-phenyl-1,4quinonediimine)(ATQD), and grafted it with bioactive peptide RGD (ATQD-RGD). This bioactive electroactive scaffold material supported adhesion and proliferation of PC-12 cell, and stimulated spontaneous neurogenesis in PC-12 cells (also in the absence of NGF and even more in its presence).

\section{Effects due to orientation, aligning, patterning, and/or size}

Lee, Schmidt et al. [52] layered aligned nanofibers, prepared by coating PPy on fibers prepared by electrospunning of copolymers of lactic and glycolic acids (PLGA). Electric surface resistance in the direction of the aligned PLGA fibers was $7.4 \pm 3.2 \times 10^{3} \Omega$ /square, and it was $14 \pm 6 \times 10^{3} \Omega$ /square on the randomly oriented fibers. PC-12 cells and embryonic hippocampal neurons were cultured on scaffolds prepared using the aligned (AF) and the randomly oriented (RF) fibers. Electrical stimulation of PC-12 cells cultured on AF conducting nanofiber scaffolds improved significantly neurite outgrowth. Greater neurite outgrowth was induced by the low electrical potential of $10 \mathrm{mV} / \mathrm{cm}$ than by the higher electrical potential of $100 \mathrm{mV} / \mathrm{cm}$. This effect was more pronounced on the AF then on the RF fibers. Gomez, Schmidt et al.[53], suggested that cell stimulation may be affected by patterning of PPy. Less defined rough structures were also produced when the PPy/dopant concentrations, electropolymerization currents, and beam intensities were high. PC-12 cells cultured for $20 \mathrm{~h}$ on the properly patterned PPy had well defined axons, as compared with only a twofold increase in their size on the unoriented PPy. In order to take full advantage of cell stimulation by patterned PPy, it may be further modified by immobilizing NGF on it. The mentioned above procedures may be advantageous for such neural tissue engineering applications as neural probes and nerve conduits.

\section{Doping PPy with biologically active molecules}

Gilmore, Wallace, et al.[54] used PPy substrates doped with ECM components, in order to check which may be used to promote differentiation and adhesion of skeletal muscle myoblasts and nerve cells. All dopant combinations supported, to some extent, the proliferation and adhesion of $\mathrm{C} 2 \mathrm{C} 12$ and myoblast cells. However, not all combinations supported the differentiation of myoblast cells and formation of myofibrils. PPy/CS, PPy/DS, and PPy/DBS (dodecyl benzene sulphate) provided good support for both proliferation and differentiation of myoblast, but PPy/HA and PPy/pTS, which showed good support for proliferation of myoblast, support poorly its adhesion and differentiation. The use of the bioactive dopants: chondroitin sulphate salt (CS), and dextran sulphate (DS) did not provide any advantage in terms of biocompatibility. Poor performance of the bioactive dopant $\mathrm{HA}$ (hyaluronic acid) in terms of cell adhesion may apparently be attributed to its hydrophilic nature. Cell adhesion and differentiation supported by PPy/PMAS poly(methyl aniline sulphonic acid) was good, but only a low degree of proliferation could be attained on this support. Kim, Martin et al. [55] incorporated neurotropic biologically active dopant such as NGF in PPy and PEDOT. The modified substrates extended neurites on both PPy and PEDOT, showing that the doped NGF was biologically active, and created significant populations of PC-12 cells. Meng, Rouabhia, et al.[56] doped PPy with heparin and incorporated the doped particles in PLLA. They found that PPy/PLLA membranes containing such particles were electrically more stable in an aqueous 
environment. Adhesion and growth of human fibroblast cells also improved on such membrane.

\section{Use of ECPs in stem cell investigations}

Bechara, Popat et al.[57] found that scaffold made of PCL nanowires coated with PPy facilitate the differentiation, proliferation, and adhesion of $\mathrm{C} 17.2$ neural stem cells.

\section{ECP blends or composites with non ECPS}

Forciniti L., Schmidt C.E., et al.[58] investigated preparation and properties of blends of poly(lactic-glycolic acid) (PLGA) with PPy, which on top of their electrical conductivity are characterized by low impedance, surface roughness, and increased toughness required for microelectrodes, used in many biomedical engineering applications. They prepared porous PLGA films, obtained by spin coating PLGA from its solutions containing either $20 \%$ or $40 \%$ of $\mathrm{NaCl}$. Subsequently electrochemical (EC) and chemical (C) approach was used. 1) EC-approach: salt was leached with water, and porous PLGA film was obtained. IT was soaked for $12 \mathrm{~h}$ with $0.1 \mathrm{M}$ solution of pyrrole and submitted to an oxidizing voltage of $0.82 \mathrm{~V}$ (vs. SCE). The resulting hybrid film of PLGA:PPy was sonicated, washed with water, and dried in vacuum for 2 days. 2) Chemical approach: An aq. solution of pyrrole+ $\mathrm{FeCl}_{3}$ was allowed to react for 12 hours with the Pyrrole soaked PLGA film. The PPy:PLGA films created by using both approaches, showed $87 \%$ decrease in impedance (effects due to orientation of PLGA-PPy composite fibers are discussed in refs.52-53 in section $2 \mathrm{c}$ of this review). Fabrication of PPy doped PLLA- $\mathrm{H}_{2} \mathrm{SO}_{4}$ was recently described by $\mathrm{Yu}$ et al.[59]. They suggested that the above described dual multiporous composite micro/nano fiber films may be suitable as scaffolds for TE of the bone or nerve cells, and for the regeneration or reconstruction of the respective tissues. They proposed to combine electrospinning with in-situ polymerization and doping. Blends of PDLA with PANI were investigated by McKeon et al.[60] Though such blends support cell adhesion and growth, their shrinkage seems to prevent their use as a primary component of biomedical devices.

Xie, Xia et al. [61] prepared core-sheath nanofibers using either PLLA or poly $(\varepsilon-$ caprolactone) PCL nanofibers as a template. They coated them with pyrrole, which they polymerized and oxidized in-situ using $\mathrm{FeCl}_{3}$. They found in the in-vitro experiments that the explanted dorsal root ganglia adhere well to such core-sheath nanofibers and neurites may be generated and oriented when NGF is added to the medium. Yaszemski and his collaborators prepared copolymers of fumaratepolypyrrole composites for the TE and nerve regeneration applications. Moroder, Yaszemski et al.[62] investigated composite of PCLF-PPY as nerve conduit for the repair of severe nerve damages. Significant increase in the number of neurites bearing PC-12 cells was observed in vitro, as results of electrical stimulation. Surface resistance of such scaffolds was $2 k^{\prime} \Omega$ and they were stable during electrical stimulation. Runge, Yaszemski et al. [63,64] used such composites, as well, as their oligo polyethylene glycol analogues for nerve regeneration. Mechanically resilient electroactive scaffold was prepared by Broda, Schmidt et al. [65]. They emulsified Py in chloroform solution of polyuretane (PU), and polymerized it by adding drop wise solution of $\mathrm{FeCl}_{3}$ in chloroform, up to molar ratio of 3 mols of $\mathrm{FeCl}_{3}$ per one mol of Py. The PPy was oxidized and doped with chlorine. The doped polymer was quenched with ethanol after 3 hours. The precipitated composite was washed with 
ethanol, pressed to $1 \mathrm{~mm}$ thickness and dried in vacuum. The PPy/PU weight ratios in various samples varied from 1:5 to $1: 100$. The resulting composite was cytocompatible with $\mathrm{C} 2 \mathrm{C} 12$ cells. Elastic, electroactive nanofibers containing polyaniline doped with camphor sulfonic acid (PANI-CMSA) were blended by Jeong, Shin et al. [66] with poly(L-lactide-co-PCL). Electrospunning of CMSA-PANI/PLCL produced smooth, homogeneous nanofibers with high pore volume of interconnecting pores. Their diameters were in the 100 to $700 \mathrm{~mm}$ range, with nearly constant mean diameter of $400 \pm 15 \mathrm{~mm}$. Their conductivities increased with the increase in the CMSA-PANI content from 0.0015 to $0.0138 \mathrm{~S} / \mathrm{cm}$, at the $30 \%$ of PANI-CMSA content. Their adhesion to NIH-3T3 human dermal fibro-blasts and to $\mathrm{C} 2 \mathrm{C} 12$ myoblasts was significantly higher than that to pure PLCL fibers. It also depended on PANI-CMSA content. Moreover, growth of NIH-3T3 fibroblasts in contact with such material may be stimulated by electric potential. Giuseppe-Eli as well as Wallace at al. [67, 68] investigated blends and co-networks of ECPs with highly hydrated hydrogels and found that they combine properties of both constituents. The electroactive component provides electric conductivity, electrochemical redox properties and on/off switching. Biocompatibility, swellibility, and high diffusivity of small molecules is provided by hydrogel component. They described synthesis and properties of poly (HEMA) based hydrogels with PANI, which enable fabrication neural prosthetic devices with low voltage actuation for drug release, deep brain penetration and biocompatibility of implantable sensors.

\section{Copolymers of PPY with not ECPS}

Experiments conducted by Durgam, Schmidt et al.[69] showed that electrical

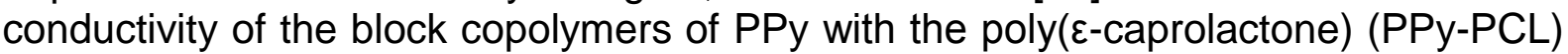
and with the poly(ethyl cyano-acrylate) (PPy-PECA) was slightly greater or slightly smaller than the PPy homopolymer; (32 S/cm for PPy-PCL, 19 S/cm for PPy-PECA, and $22 \mathrm{~S} / \mathrm{cm}$ for the PPy homopolymer). PPy component electrically stimulated neural cells growth and proliferation, while the other copolymers enabled their degradation and hydrolysis. In subsequent experiments these copolymers were coated into the inner walls of the nerve guiding channels made of the biodegradable polymer poly(3-hydrobutyrate-co-3-hydovalerate). The guiding channels were implanted into sciatic nerve of rats with $10 \mathrm{~mm}$ defects (made in them for this study). Histological staining showed axonal growth, when they were harvested after 8 weeks. Guimard, Sessler, Schmidt [70] investigated copolymers containing oligothiophenes as electroactive units. They contained the biodegradable adipic acid polyester with intermittent bis(hydroxymethyl)-dimethyl-quarter-thiophene (APE-coQTn). It was prepared via polycondensation reaction. The copolymer was biocompatible, non toxic, and moderately electroactive.

\section{Chemically modified electroactive polymers}

Lee J.Y., \& Schmidt [71] coupled Py with hyaluronic acid (HA). Such coupling made the adduct biocompatible. Hyaluronic acid prevented adhesion and migration of fibroblast and astrocytes towards the coated surface. Such coating of electrodes enabled stable measurements under physiological conditions. A carboxyl end-caped PPy was investigated by Lee, Serna, Schmidt [72] They modified surface of PPy by a carboxylic acid group by attachment of a pyrrole-a- $\mathrm{COOH}$ molecule. The modified polymer retained its mechanical integrity, and its electroactive properties. Moreover the carboxylated surface could be modified further. 


\section{Other electroactive polymers}

Bettinger, Langer et al. [73] investigated the use of melanin for nerve regeneration. Melanin is a naturally occurring electrically conducting pigment, in vivo and in vitro biodegradable. Though its exact chemical structure is still not known it is believed to be an extended heteropolymer of dihydro-indole and its carboxylic acid derivative. They prepared thin molecularly smooth melanin films with electric conductivity, in the hydrated state, of $7.00 \pm 1.1 \times 10^{-5} \mathrm{~S} / \mathrm{cm}$. They can be made as thin as $4 \mathrm{~nm}$. They are nontoxic and biodegradable in vivo and in vitro. They enhance Schwann cell growth and neurite extension when inserted in their proximity. Such films were found, after 8 weeks, to be nearly fully in vivo degraded. Authors believe that their results suggest that melanin may be used for tissue engineering applications. Zhang et al.[74] thermally polymerized hexachlorocyclotriphosphazene and substituted (by nucleophilic substitution) one set of chlorine atoms of the poly(dichlorophosphazene) by ethyl ester of glycine $(n=16000)$ and another set by aniline pentamer. The polymer formed was doped with camphor sulfonic acid (CMSA). Its semiconductive properties depended on the degree of doping. It was found to be biocompatible and to promote in vitro proliferation of RSC96 cells. Authors believe that PGAP has a potential as neuronal TE scaffold material.

\section{ECPs for drug targeting and delivery}

\section{Release and diffusion}

Niamlang \& Sirivat [75] investigated the apparent diffusion coefficients, $\left(D_{\text {app }}\right)$, of salicylic acid (SA) (as a model drug) doped into poly(p-phenylene vinylidene)[PPV], as well as mechanism of its release from PPV blended with polyacrylamide (PAAm) hydrogel. They compared it with the release of SA from PAAm hydrogel loaded with $\mathrm{SA}$. Measurements were conducted in the presence and in the absence of an external electric field of $0.1 \mathrm{~V}$, which was applied either to anode or to cathode terminals. For the first $3 \mathrm{~h}, \mathrm{SA}$ was not released at all from the doped by it PPV, without electric stimulation. Afterwards it started to diffuse through the PPV/PAAm blend until equilibrium concentration was reached. When the electric field was applied to the anodic terminal, SA was initially not released by doped with it PPV, because ionic interaction between conductive polymer and SA counter ions prevented it. On the other hand, when the potential was applied to the cathode, the release was very pronounced and increased with the increase in the strength of electric field. The release of SA loaded into PAAm hydrogel was also affected by the applied current. However $D_{\text {app }}$ of $S A$ diffusing from PAAm hydrogel was smaller by one order of magnitude than $D_{\text {app }}$ of $S A$ released by SA-PPV. Chansai, Sirivat et al.[76] prepared poly(acrylic acid) (PAA) hydrogel, crosslinked by ethylene glycol dimethacrylate (EGDMA). Various degree of crosslinking was obtained by changing the proportion of EGDMA. Crosslinked PAA hydrogels were blended with PPy doped with 5-sulfosalicylicylic acid (SSA) (as a model drug). Diffusion of SSA through the PAA hydrogels was compared with their diffusion through the matrices of the conductive blends. When the degree of crosslinking was decreased and the strength of the applied electric field was increased, the diffusion coefficients increased. Investigators concluded that PPy promotes transport of SSA through PPA matrix, and its rate of release can be controlled by adjustment of the appropriate parameters. Cho et al.[77] investigated mesoporous silica nanoparticles ( 100 nm in diameter) 
loaded with NGF and imbedded into PPy film. This system was able to control release of NGF, which induced specific cellular response in the PC-12 cells targeted by it. Their spreading, adhesion and proliferation were significantly increased. These effects were strongly enhanced by electric stimulation of the PPy film. Lu \&Cui [78] investigated the electrically controlled drug release from the sponge-like nanostructured PPy film. It was found that the drug can be loaded not only into the bulk of PPy, but also onto its nano holes. The choice of molecules that can be loaded into the holes is not limited rigorously by their size or charge. Such system allows simultaneous release of two kinds of drugs, which may be required for a given treatment. For example for simultaneous delivery of enzyme and is cofactor. Application of ECPs for controlled release and delivery of Dexamethasone DEX (antiinflammatory drug which reduces inflammation in the central nervous system) was described and discussed in refs.79-82. Wadhwa. Cui, et al. [79] electrochemically controlled release of DEX form PPy coated electrode. $0.5 \mu \mathrm{g} / \mathrm{cm}^{2} 0.5 \mu \mathrm{g} / \mathrm{cm}^{2}$ of DEX was released during each cycle of cyclic voltammetry $(\mathrm{CV})$. A total of $16 \mu \mathrm{g} / \mathrm{cm}^{2}$ of DEX could thus be released after $30 \mathrm{CV}$ cycles. In vitro studies of murine glial cells showed that the number of reactive astrocytes could thus be reduced without any toxic side effects. Authors believe that this kind of system can also be used for local delivery of other drugs and agents for treatment of injuries and disorders. Abidjan, Kim, and Martin [80] prepared conductive nanotubes coated on neural electrodes by electrospunning the biodegradable poly (L-lactic acid) or poly(lactic-co-glycolic acid) (PLGA) as a template in which DEX was incorporated. This step was followed by an electrochemical deposition of PPy or PRDOT doped with saline solution. DEX could be released after biodegradation or dissolution in chloroform of PLA, Alternatively the core of the polymer could be removed by soaking in $\mathrm{CH}_{2} \mathrm{Cl}_{2}$ and the conductive tubes (containing DEX inside it) could be deposited on the tips of the microelectrodes. DEX could be delivered by activating the microelectrodes. (This step was possible thanks to the decrease of impedance of recording sites as the result of coating them with PEDOT or PPy). Stevenson, Wallace, et al. [81] investigated polyterthiophene (PTT) as an electro-stimulated material for the release of DEX. Disodium phosphate of DEX can be released from PTT at therapeutically relevant levels. The oxidation state of PTT is critical for the release. After applying oxidizing potential of $+0.6 \mathrm{~V}$, DEX release is reduced by $50 \%$ over $24 \mathrm{~h}$ periods. DEX doped PTT is auto reduced when placed PBS solution. No statistical difference was observed between autoreduced and electrochemically reduced PTT. Sirivisoot, Pareta, and Webster [82], released DEX and/or penicillin/streptomycin (P/S) in a controlled fashion, from PPy immobilized on an anodized nanotubular Titanium (MWNT-Ti). Pyrrole monomers were oxidized by P/S or by DEX (incorporated as anionic dopants). Cyclic voltammetry (CV) was used to stimulate the release of drugs from PPy. Results of CV showed that $80 \%$ of drugs could be released on demand by applying 5 cycles of voltages sweep at a scan rate of $0.1 \mathrm{~V} / \mathrm{s}$. This investigation indicated that controlled release of these drugs improved bone implants. They enhanced adhesion of osteoblasts and inhibited fibroblast (scar-tissue cells) formation. Stauffer, Cui et al. [83] modulated local neural activity by controlled drug release from PPy coated on recording microelectrodes. However, without an external reservoir the number of molecules that can be thus released was quite limited. These investigators attempted to increase drug load, to attain identical release at each pulse applied, and to increase its efficiency, by creating a nanostructure polymer film. They attempted to optimize stimulation drug release, and conditions of polymer synthesis, in order to attain maximal, identical release at each pulse applied. Moreover, electroactive 
polymers, other than PPy, could also be used for the electrochemically controlled release of various agents. Kang, Borgens and Cho [84] deposited PPy electrochemically on colloidal polystyrene arrays, which served as a template for the formation of a well ordered porous PPy doped with NGF and sodium salt of poly(styrene sulfonate) (NaPSS). They deposited, under constant catholic potential of $0.9 \mathrm{~V}$ aqueous mixture of $0.1 \mathrm{M}$ pyrrole, $0.1 \mathrm{M} \mathrm{NaPSS}$ and $100 \mu \mathrm{g} / \mathrm{ml}$ of NGF. The resulting porous film of doped PPy was dried and stored in vacuum. The PPY-NGF doped film was incubated at $37^{\circ} \mathrm{C}$ in PBS solution and $0.1 \mathrm{~V}$ potential applied for $2 \mathrm{~h}$ in order to release NGF. The release profile of NGF doped into the open structure porous PPy increased dramatically in comparison to NGF doped onto a flat PPy sheets, especially when it was stimulated electrically. Cell adhesion, metabolic activity and neurite extension of cells cultured on the porous PPy was superior to those, which were cultured on flat PPy sheets. Cho and Borgens [85] prepared biotin doped porous PPY, using a procedure, which was in principle analogous to the procedure described in ref. $84.9 \mathrm{mM}$ of Biotin and $0.01 \mathrm{M} \mathrm{NaDBS}$ were used instead NGF and NaPSS, and the applied potential was $0.7 \mathrm{~V}$ not $0.9 \mathrm{~V}$. When the biotin doped PPy was exposed to a suspension of gold-streptavidin (Au-Sa), they absorbed gold particles as result of the biotin-streptavidin interaction. The absorbed Au-Sa nanoparticles can be released in a controlled fashion by applying negative positive potentials ranging from 1 to $2 \mathrm{~V}$. When $+2 \mathrm{~V}$ potential was used all the nanoparticles were released within $60 \mathrm{sec}$. This experiment demonstrated that biotin doping combined with biotin-streptavidin interactions can be used for controlled release of many important biologically substances. Shepherd \& Wallace [86] achieved autonomously controlled drug release due to galvanic coupling between an electrode (I) immersed in PBS solution at $\mathrm{PH}=7,2$ and biodegradable $\mathrm{Mg}$ alloy attached to it (which dissolves during the release of a drug to form $\mathrm{Mg}^{2+}$ ions ) and Electrode (II) connected to It, enveloped by a film of conductive polymer doped with a drug to be released, and immersed in an identical PBS solution. The rate of drug release may be adjusted by changing the thickness and/or porosity of the coating, method of preparation or the type of the conducing polymer. Majumdar et al.[87] applied a mathematical framework of the dynamics and performance involved in the molecular drug release of a given drug from an ECP, in order to calculate parameters of its release. A huge set of simulations varying in operational parameters and design was considered. Authors believe that system developed by them will enable optimization of a wide range of therapeutic applications of ECPs for controlled drug release and delivery.

\section{Targeting and delivery}

Wuang, et al. [88] synthesized nanoparticles of $\mathrm{PPy}$ with $\mathrm{Fe}_{3} \mathrm{O}_{4}$ encapsulated in them by emulsion polymerization (with PVA as surfactant). The size (80-100 nm) and shape of the $\mathrm{PPy}-\mathrm{Fe}_{3} \mathrm{O}_{4}$ nanoparticles was well defined. Their magnetization values were $>20 \mathrm{emu} \mathrm{g}^{-1}$ and electric conductivity was ca. $1 \mathrm{~S} / \mathrm{cm}$. At $\mathrm{Fe}_{3} \mathrm{O}_{4}$ content of $28 \%$, they were well dispersed and did not show any significant toxicity. Surfactant's functional groups allowed immobilization of folic acid or other biomolecules. Folic acid bonded to them targeted efficiently MCT-7 human breast cancer cells, as a result of the receptor induced endocytosis and possibly also some non specific binding. It is hoped that these nanoparticles will serve as cancer cell-targeting agents and as intracellular seeds in cancer treatment therapy. George, Langer et al.[89] proposed to attach drug to be delivered to biotin molecules, which were attached as dopants to PPy. In this way they circumvented problems related to the choice of 
dopant and were not limited by the molecular weight of the drug. They investigated neurite outgrowth from PC-12 cells cultured on PPy doped with biotin to which NGF molecules were attached through streptavidin links. Stimulation of PP by the $3 \mathrm{~V}$ potential was applied at $24^{\circ} \mathrm{C}$ across BPS solution. Abidian, Kim and Martin [90] reported that DEX loaded into PLGA tubes surrounded by PEDOT could be delivered at a given moment by electric pulse, which caused contraction of PEDOT surrounding PLGA containing DEX and pushed part of the drug into surrounding solution or blood.

\section{ECPs as sensors of biologically important molecules}

The use of the electrically conducting polymers (ECP's) for biosensors is related to the assessment of their role as a reporting interface. Biosensors can operate under harsh conditions in an organic phase and they are expected to produce microelectronics' sensing devices. Electrochemical (EC) biosensors [91, 92] based on CP's may provide immobilization of biomolecules and rapid electron transfer. In biomedical applications their use often involves incorporation of anionic biological species such as proteins and polysaccharides. They can operate [93] as electrochemical sensors, tactile sensors, which respond to changes in pressure, force, or touch, and as thermal sensors, which respond to changes in temperature. Tremendous advances in clinical testing have been achieved in recent years thanks to their application. Papers recently published on this subject are listed in sections $4 a$ and $4 \mathrm{~b}$ of this review. Tactile sensors- based on ECPs may be applied in medicalrobotics and telemedicine. They are not discussed in the current review.

\section{Nucleic acid electrochemical (EC) sensors}

DNA sensors combine nucleic acid layers with EC transducers to produce a biosensor. They provide a simple, accurate and inexpensive platform for patient diagnosis. They rely on specific recognition events for detection of a target analyst. The sensor provides a platform that facilitates the formation of a probe-target complex whose binding triggers the signal for electronic readout. The signal transducer may be coupled to a readout device [94]. The base pairing interactions between the complimentary sequences of DNA are specific and robust. Hence, it is especially well suited for such bio-sensing applications. DNA sensors combine nucleic acid layers with EC transducers to produce a biosensor. They provide a simple, accurate and inexpensive platform for patient diagnosis. They rely on specific recognition events for detection of a target analyst. The sensor provides a platform that facilitates the formation of a_probe-target complex whose binding triggers the signal for electronic readout. The signal transducer may be coupled to a readout device [94]. The base pairing interactions between the complimentary sequences of DNA are specific and robust. Another problem is due to the enormous biological complexity of genomic DNA samples. The sensitivity of the EC detection could be insufficient for detection of mismatched base pairs. Therefore, a round of PCR (polymerase chain reactions) or other biochemical multiplication could sometimes be required. The paper published by Wei, Lillehoj \& Ho [95] described a very high sensitivity nanotechnology-enhanced EC detection of nucleic acids that enables detection of mismatched base pairs in DNA, and enables treatment in the early stages of genetically-related diseases and conditions The EC sensors have high sensitivity and specificity essential for detection of a mismatch of a single base pair. These researchers pointed out that the recent advances in the micro- and 
nanotechnologies contribute to the emerging potential of EC DNA diagnostics. A variety of new materials and fabrication processes is involved in these technologies. Detection sensitivity was enhanced through highly specific molecular recognition, by improved generation of the EC signals, transduction and amplification, enhanced electrical conductivity and minimized background noise. Sensors are also exceptionally efficient in terms of fast detection times and low power consumption. Contributions from microfluidic and MEMS fabrication and micromachining make possible relatively simple handling on portable diagnostic platforms. Cagnin, Lanfranchi et al.[96] published in 2009 an overview of the EC DNA Biosensors. They indicated that DNA micro-arrays were created to investigate large numbers of characterized genes. Single nucleotide polymorphism was characterized to identify the in vivo transcription factor (TF), binding sites, and chromosome deletion or amplification. DNA biosensors have the potential to overcome the limits of the DNA micro-arrays by providing rapid and highly sensitive analytical tools for genetic detection. Some currently available types of biosensors have been schematically depicted by these investigators in Table II of their review. One must be aware of various problems that may be caused by their applications, but those based on the electrochemistry are particularly promising because of their sensitivity and miniatererization opportunities, provided by the construction of the CombiMatrix sensor, a very large multiplex array. Lucerelli, Marrazza et al.[97] described genotyping based on the use of split hybridization probes and developed a highly selective single-nucleotide polymorphism (SNPs) typing method through EC analysis of single-base mutations in actual patients samples. Their specific probe design, hybridization, and signal transduction path simplified the analytical process, making it suitable for low throughput analysis of clinically relevant samples. Galndova \& Labuda [98] cite in Table II of their review paper, names, preparation, transduction, and application of polymers and their nanocomposites used for EC DNA biosensors, as well as for the study of DNA sequences and their chemical interactions. References to relevant papers, most of them published in recent years, have also been provided in their review. Cleric-Pederson, Roziosnik et al.[99] developed tosylate doped PEDOT biosensor electrodes for fast detection of virus in human cell cultures. The use of such sensors may reduce significantly time required for virus diagnosis. Fan, Gao, et al.[100] described ultrasensitive detection of micro-RNA with the target-guided deposition of PANI nanowires. The method directly utilized chemical legation and amplification of the signal read-out, which simplified the detection procedure. They suggest that it will be possible to achieve multiplex detection by introducing different detection probes into the biosensor array. Shang, Zheng, et al. [101] developed an improved essay for EC bio sensing of syphilis DNA by using biocatalytic amplification and biotin-streptavidin binding ability for deposition of PANI template on hybridized DNA. Specificity of the biosensor was significantly improved by the design of the thiol modified capture probe and biotinylated signalling probe complementary to target regions flanked by PCR primers. Fan, Gao et al.[102] detected famtomolar quantities of MicroRNA (miRNA), which are adsorbed inside the $30 \mu \mathrm{m}$ gaps of the special gold electrodes. They were hybridized through interaction between their anionic phosphate groups and the cationic groups of the conductive PANI. The conductance of electrodes, with gaps filled in such fashion, was directly related to miRNA content. The detection limit of such a device was $5 \mathrm{fM}$ with qualified range of $10 \mathrm{fM}-20 \mathrm{pM}$. 
Xie, Yu et al.[103] fabricated the poly(EDOT-COOH) nanowire devices and modified it by attaching to its carboxylic groups the thrombin-binding aptamer: 5-T6 G TGG GT TGG TGT GGT TGG-3', 28-mer nanocomplementary probe: 5'-T6T TGA GTC TGT TTG GTC AGC-3', and 49-mer nanocomplementary probe: 5-T6T ATC TAC GGA TTC ATC AGG GTC AAA GAG TGC AGA GTT ACT TAG-3'. They demonstrated, using this device, a label-free detection of the blood-clotting factor thrombin. Substantial decrease in the current flow due to exposure to the sensor device due to its exposure to the thrombin solutions is due to a specific interaction between thrombin and aptamer-conjugated polymer chains. The dynamic range between $1 \mathrm{~nm}$ to $1 \mu \mathrm{m}$ is wide enough to cover the entire range. The PEDOT nanowire-based sensing platform can be extended to other proteins, for which DNA aptamers are available. Hafaid et al. [104] described fabrication and properties of an immunosensor based on immobilization of HIS-tag antibody on an electropolymerized co-PPy film coordinated with the NTA/copper complex attached to a gold electrode. Specific antigen detection obtained by electrochemical impedance spectroscopy (EIS) was in the 0.1 to $10 \mathrm{ng}^{-1} \mathrm{ml}^{-1}$ range, its detection limit was 0.1 ng. $\mathrm{ml}^{-1}$. Tully et al.[105] developed an EC immunosensor for direct detection of protein at the pictogram level. PANI was laid down, as thin-film layer, on electrode surface. Antibody (Internalin B) was immobilized on PANI. It was coupled to sensor's matrix through neutravidin - biotin. Impedimetric measurements at the lower end of frequency range of $1-10 \mathrm{~Hz}$ revealed an overall impedance change due to specific interactions of the complexes formed. The lower limit of detection was $4.1 \mathrm{pg} / \mathrm{ml}$. Authors believe that this sensor provides a promising tool for label-less detection of InIB F3. Lee, Yun et al.[106] fabricated PANI single nanowire sensor by electropolymerization of ionized aniline in a nano-channel $100 \mathrm{~nm}$ wide built lithographically in gold electrodes. Distance between the 2 electrodes was 5microns. PANI was immobilized with anti-rabbit IgG and mAbs protein. This biosensor showed specificity and sensitivity towards $\lg G$ and Myo. It can be also used for detection of other mAbs of various proteins.

\section{Amperometric sensors of glucose and other sugars}

Gade, Gaikwad, Shirsat et al.[107,108] prepared PPy-PVS-GOD or PANI-PVS-GOD amperometric enzyme sensors of glucose by immobilizing glucose oxidase (GOD) on PPY or PANI films doped by PVS \{poly(vinyl sulfonate)\}. GOD enzyme was immobilized, through cross-linking with glutar-aldehyde. Such films were synthesized electrochemically by galvanostatic deposition technique from pyrrole monomer or aniline and doping it with PVS. ITO glass plate was used as a working electrode on which Py or aniline were polymerized and PVS was deposited and into PPy or into PANI films. Thus prepared electrodes had short response time, long life time, and have dynamic range of detection of glucose. Arslan et al.[109] prepared PANI-PVSGOD amperometric sensor of glucose, analogous to that described in ref 108 by using Pt as a working electrode instead of the ITO coated glass plate. Yu \& Sundmacher [110] used ferrocene for oxidation and doping of PPy for the preparation of analogues glucose sensors. Şenel [111] prepared recently analogous glycol sensors by condensing Py with Py-ethanol and carboxyethyl-Py, electro-polymerizing it on a glassy carbon electrode and immobilizing on it GOD, and Ferrocene through its hydroxyls, and carboxylic groups, respectively. Its detection limit was found to be $6.9 \mu \mathrm{M}$ of glucose. A glucose sensor with linear response in the 1,1-16.5 mM range of 
glucose prepared by immobilizing GOD on PEDOT doped by polystyryl sulfonate (PSS) was constructed by Liu Varahramyan et al. [112]. The Field-Effect-Transistor (FET) sensor of glucose providing reproducible response in 0,5-20 mM concentration range was fabricated by Yoon, Jang et al. [113] using polypyrrole carbonate, enzyme functionalized, tubes as a conductive channel. A generic paradigm for construction of integrated enzyme electrodes with effective electrical contacts was formulated by Willner et al.[114]. They showed that it can be achieved by electropolymerization of the electropolymerizable Au nanoparticles (NPs) together with electropolymerizable GOD. They showed that could be achieved by: 1) attachment of the Au NPs to 4-mercaptoaniline and mercaptoethane sulfonic acid; 2) functionalization of GOD through reaction with lysine residue attached to $\mathrm{N}$ (maleimidocaproyloxy) sulfosuccinimide ester, followed by reaction with 4mercaptoaniline. The effective electric contact of the enzyme with the electrode in such system facilitates the detection of glucose in the presence of $\mathrm{O}_{2}$. These investigators believe that this approach will be useful for fabrication of tailored, miniaturized electrocatalytically active electrodes. Lee, Son et al. [115] increased sensitivity of galactose biosensors by fabricating them in a bundle of conductive tubules, which was prepared by electrochemically polymerizing EDOT into template on ITO. Tubules provided larger enzyme encapsulating space per unit area than other structures. The average sensitivity was $6.37 \mu \mathrm{A} / \mathrm{mMcm}^{2}$, the detection limit was $.01 \mathrm{mM}$ and response time $30-40 \mathrm{sec}$.

ECPs for the determination of the concentration of $\mathrm{H}_{2} \mathrm{O}_{2}$

Chen, Yuan et al. [116] used AuNPs, chitosan, and HRP (as catalyst for oxidation of chitosan by $\mathrm{H}_{2} \mathrm{O}_{2}$ ) on PANI fibers. The hybrid film were located on a Pt electrode, which was immersed in $0.1 \mathrm{M}$ PBS solution $(\mathrm{pH}=6)$. Potential of $0.35 \mathrm{~V}$ was applied. Known amounts of $\mathrm{H}_{2} \mathrm{O}_{2}$ were introduced into the solution at $30 \mathrm{sec}$ intervals and corresponding currents were recorded. This biosensor was characterized by good reproducibility, long-term stability, rapid linear response to $\mathrm{H}_{2} \mathrm{O}_{2}$ in the concentration range of $7 \times 10^{-6}-1.4 \times 10^{-2} \mathrm{M}$, and detection limit of $2.8 \times 10^{-6} \mathrm{M}$. Lupu et al.[117] prepared an essentially analogous sensor by using PEDOT instead of PANI and Prussian blue instead of chitosan. However, The detection limit of the proposed by them sensor was only $10 \mu \mathrm{M}$ with linear range from $1 \times 10^{-5}$ to $1 \times 10^{-4} \mathrm{M}$.

\section{Acknowledgements}

This research is made possible in part by the historic generosity of the Harold Levin family.

\section{References}

[1] Pratt, C. http://homepage. nt/world.com/colin.pratt/app/cp.html.

[2] Bolto, B.A.; Mc Neill, R.; Weiss, D.E. Australian J. Chem. 1963, 16, 6, 1090.

[3] McNeill, R.; Weiss, D.E.; Willis, D. Australian J. Chem. 1965, 18, 477.

[4] Bolto, B.A.; Weiss, D.E.; Willis, D. Australian J. Chem. 1965, 18, 487.

[5] Seymour, R.B. (ed.), Conductive Polymers. Plenum Press: New York, 1981 p.2347.

[6] Shirakawa, H.; Ikeda, S. Polymer J. 1971, 2, 231.

[7] Shirakawa, H.; Ito, T.; Ikeda, S. ibid 1973, 4, 460.

[8] Shirakawa, H.; Ito, T.; Ikeda, S. Kobushi Rombushi ,1976, 33, 339.

[9] Ito, T.; Shirakawa, H.; Ikeda, S. Polym. Chem. Ed. 1975, 13, 1943. 
[10] Shirakawa H., Louis E.J., MacDiarmid A.G., Chiang C.K., Heeger A.J.,.J. Chem. Soc. Chem. Commun. 1977, 16, 578..

[11] Enkelmann, V.; Muller, W.; Wegner, G. Synth. Metals 1979/80, 1, 185.

[12] Jagur-Grodzinski, J. Polymers Adv. Technol. 2002, 13, 615.

[13] Heeger, A.J.; MacDiarmid, A.G. Chem. Scripta 1981, 17, 115.

[14] Ikheta, S.; Kaufer, J.; Pron, A.; Woerner, T.; Druy, M.A.; Sivek, A. Phys. Rev.Lett.1980, 45, 1123.

[15] Suzuki, N.; Jr., Ozaki, M.; Etemad, S.; Heeger, A.J.; MacDiarmid, A.G. Ibid 1209.

[16] Mele, E.J.; Rice, M.J. Ibid, 926.

[17] Kao, C,Y.; Lee, B.; Wielunski, L.S.; Heeney, M.; McCoulloch, I.; Garfunkel, E.;

Feldman, L.C.; Pozdorow, V. Adv. Funct. Mater. 2009, 19, 1906.

[18] Frommer, J.E.; Chance, R.R. Encycl. Polym. Sci Eng. 1986, 5, 462.

[19] Thakur, M. Macromolecules 1988, 21, 661.

[20] Shang, Q-Y.; Pramanick, S.; Hudson, B. Macromolecules 1990, 23, 1886.

[21] Shopow, I.; Sinigerski, V. Makromoleculare Chem. 2003, 193, 1839

[22] Bakhshi, A.K.; Bhalla, G. Industrial Res. 2004, 63, 715.

[23] Aldisi, M. Synth. Met. 1984, 9, 131.

[24] Sathiyanarayanan, S.; Azim, S.S.; Venkatachari, G. Synth. Met. 2007, 157, 205.

[25] Trueba, M.; Trasatti, S.P. Prog. Organic Coating 2009, 66, 265.

[26] Hosseini, M.G.; Raghibi-Boroujeni, M.; Ahadzadeh, I.; Najjar, R.; Dorradji, M.S. ibid, 321.

[27] Narayanasamy, B.; Rajendram, S. Prog. Org. Coat. 2010, 67, 246.

[28] Lu, W-K.; Basak, S.; Elsenbaumer R.L., Corrosion inhibition of metals by conductive polymers, p.885 in Handbook of Conductive Polymers, II Eds. Skotheim, T.A.; Elsenbaumer, R.L.; Reynolds, J.R. Marcel Dekker, Inc. 1998. New York, Basel. [29] Bandera, A-D.; Cianga, L.; Cianga, I. J. Biomat. Appl. 2011, 26, 3.

[30] Kulkami, V.C. Transparent conductive coatings, p.1068 in chap.38 of ref. 28.

[31] Kotov, N.A.; Winter, J.O.; Clements, I.P.; Jan, E.; Timko, B.P.; Campidelli, S.; Pathak, S.; Mazzatenta, A.; Lieber, C.M.; Prato, M. et al. Advanced Mater. 2009, 21, 3970.,

[32] Guimard, N.K.; Gomez, N.; Schmidt, C.E. Prog. Polym. Sci. 2007, 32, 876.

[33] Wan, Y.; Yu, A.; Wu, H.; Wang, Z.; Wen, D. J. Mater. Sci. Mater. Med. 2005, 16, 1017.

[34] Bhadra, S.; Khastgir, D.; Singha, N.K.; Lee, J.H. Prog. Polym. Sci. 2009, 34, 783.

[35] Chan, G.; Mooney, D.J. Trends Biotechnol. 2008, 26, 382.

[36] Barriere, F.; Mahmood, T.A.; de Groot, K. Mater. Sci. Eng. R 2008, 59, 38.

[37] Straley, K.S.; Foo, C.W.O.; Heilshorn, S. J. Neurotrauma 2010, 27, 1.

[38] Okazuki, H.; Suzuki, H.; Ito, T. Synth Metals 2009, 159, 2233.

[39] Smella, E. MRS Bulletin, 2008, 33,197.

[40] Wang, X.; Gu, X.; Yuan, C. S.; Zhang, P.; Zhang, T.; Yao, J.; Chen, F.; Chen, G. Biomed. Mater. Res. A 2003, 78A, 411.

[41] Bayer, C.L.; Trenchant, I.J.; Pappas, N.A. J. Biomater Sci.-Polym. Ed. 2010, 5, 423.

[42] Fonner, J.M.; Schmidt, C.E.; Ren, P. Polymer 2010, 51, 4985.

[43] Yu, L.M.Y.; Leipzig N.D.; Shoichet, M.S. Materials Today, 2008, 11, 36.

[44] Gomez, N.; Schmidt, C.E. J. Biomed. Mater. Res. 2007, 81A, 135.

[45] Lee, J.Y.; Lee, J.W.; Schmidt, C.E. J. R. Interface 2010, 30, 801.

[46] Kotwal, A.; Schmidt, C.E. Biomater. 2001, 22, 1055.

[47] Akkouch, A.; Shi, G.; Zhang, Z.; Rouabhia, M. J. Biomater. Res. 2010, 92A, 221. 
[48] Thompson, B.C.; Richardson, R.T.; Moulton, S.E.; Evans, E.J.; O'Leary, S.O.; Clark, G.M.; Wallace, C.G. J. Controlled Release, 2010, 141, 161.

[49] Richardson, R.T.; Wise, A.K.; Thompson, B.C.; Flynn, B.O.; Atkinson, P.J.; Fretwell, N.J.; Fellon, J.B.; Wallace, C.G.; Shepherd, R.K.; Clark, G.M.; O'Leary, S.J. Biomater. 2009, 30, 2614.

[50] Huang, H. J.; Lu, L.; Ye, Z.Q.; Luo, z . J. Biomed. Mater. Res. 2010, 93A, 164.

[51] Guo, Y.; Li, M.; Mylonakis, A.; Han, J.; MacDiarmid, A.G.; Chen, X.; Lelkes, P.I.; Wei, Y. Biomacromol. 2007, 8, 3025.

[52] Lee, J.Y.; Bashur, C.A.; Goldstein, A.S.; Schmidt, C.E. Biomater. 2009, 30, 4325.

[53] Gomez, N.; Lee, J.Y.; Nickels, D.; Schmidt, C.E. Adv. Functional Mater. 2007, $17,1645$.

[54] Gilmore, K.J.; Kita, M.; Han, Y.; Gelmi, A.; Higgins, M.J.; Moulton, S.E.; Clark, G.M.; Capsa, R.; Wallace, G.G. Biomater. 2009, 30, 29, 5292.

[55] Kim, D-H.; Richardson-Burns, S.M.; Jeffrey, L.; Hendricks, C.S.; Martin, D.C. Adv. Functional Mater. 2007, 17, 79.

[56] Meng, S.; Rouabhia, M.; Shi, G.; Zhang, Z. J. Biomed.Mater. Res. 2008, 87A, 332.

[57] Bechara, S.; Wadman, L.; Popat, K.C. Acta Biomaterialia 2011, 7, 2892.

[58] Forciniti, L.; Guimard, N.K.; Lee, S.; Schmidt, C.E. J. Mater. Chem. 2010, 20, 8865.

[59] Yu, Q-Z.; Dai, Z-W.; Lan, P. Mater. Sci. Eng. B 2011, 176, 913.

[60] McKeon, K.D.; Lewis, A.; Freeman, J.W. J. Appl. Polym. Sci., 2010, 115, 3,1566.

[61] Xie, J.; Mac Evan, M.R.; Willerth, S.M.; Li, X.; Moran, D.W.; Sakiyama-Elbert, S.E.; Xia, Y. Adv. Funct. Mater 2009, 19, 2312.

[62] Moroder, P.; Runge, M.B.; Wang, H.; Ruesink, T.; Lu, L.; Springer, R.J.; Windebank, A.J.; Yaszemski, M.J. Acta Biomaterials. 2011, 7, 944.

[63] Runge, M.B.; Dadsetan, M.; Baltrusaitis, J.; Knight, A. M.; Ruesink, T.; Lazcano, E.A.; Lu, L.; Windebank, A.J.; Yaszemski, M.J. Biomaterials, 2010, 31, 5916.

[64] Runge, M.B.; Dadsetan, M.; Baltrusaitis, J.; Ruesink, T.; Lu, L.; Windebank, A.J.; Yaszemski, M.J. Biomacromolec. 2010, 11, 2845.

[65] Broda, C.R.; Lee, J.Y.; Sirivisoot, S.; Schmidt, C.E.; Harrison, B.S. J. Biomed. Mater. Res. A, 2011, 98A, 509.

[66] Jeong, S.I.; Jun, D. I.; Choi, M.J.; Nho,Y.C.; Lee, Y.M.; Shin, H. Macromolec. Biosci. 2008, 8, 627.

[67] Guiseppi-Elie, A. Biomater. 2010, 31, 2701.

[68] Small, C.J.; Too, C.O.; Wallace, G.G. Polym. Gel Networks 1997, 5, 3, 251.

[69] Durgam, H.; Sapp, S.; Deister, C.; Khaing, Z.; Chang, E.; Luebben, S.; Schmidt, C.E. J. Biomater. Sci. Polym. Ed. 2010, 21, 1265.

[70] Guimard, N.K.E.; Sessler, J.L.; Schmidt, C.E. Macromol. 2009, 42, 2, 502.

[71] Lee, J.Y.; Schmidt, C.E. Acta Biomateria, 2010, 6, 4396.

[72] Lee, J.Y.; Serna, F.; Schmidt, C.E. Langmuir, 2006, 22, 9816.

[73] Bettinger, C.; Bruggeman, J.P.; Misra, A.; Borenstein, J.T.; Langer, R. Biomater., 2009, 30, 3050.

[74] Zhang, Q.; Yan, Y.; Li, S.; Feng, T. Mater Sci. Eng. C. 2010, 30, 160.

[75] Niamlang, S.; Sirivat, A. J. Pharmaceut. 2009, 371, 126.

[76] Chansai, P.; Sirivat, A.; Niamlang, S.; Chotpattananont, D.; Viravaidya-Pusuvat, K.. Internat. J. Pharmaceut. 2009, 381, 25.

[77] Cho, Y.; Shi, R.; Ivanisevic, A.; Borgens, R.B. Nanotech., 2009, 20, \#275102. 
[78] Luo, X.; Cui, X.T. Electrochem. Commun. 2009, 11, 1956.

[79] Wadhwa, R.; Lagenaur, C.F.; Cui, X.T. J. Control. Release 2006, 110, 531.

[80] Abidian, M.R.; Kim, D-H.; Martin, D.C. Adv. Materials 2006, $18,405$.

[81] Stevenson, G.; Moulton, S.E.; Innis, P.G.; Gordon, G.; Wallace, G.G. Synth. Metals, 2010, 160, 1107.

[82] Sirivissot, S.; Pareta, R.; Webster, T.J. Nanotechnology, 2011, 22, 101.

[83] Stauffer, W.R.; Lau, P-M.; Bi, Q-B.; Cui, X.T. J. Neural Eng. 2011, 8, 044001.

[84] Kang, G.; Borgens, R.B.; Cho, Y. Langmuir, 2011, 27, 6179.

[85] Cho, Y.; Borgens, R.B. Langmuir, 2011, 27, 6316.

[86] Shepherd, L.; Wallace, G.G. J, Mater. Chem. 2008, 18, 3608.

[87] Majumdar, S.; Kargupta, K.; Ganguly, S.; Ray, P. Polymer Eng. Sci. 2011, 51,10, 2001.

[88] Wuang, S.C.; Neoh, K.G.; Kang, E-T.; Pack, D.W.; Leckband, D.E. J. Mater. Chem. 2007, 17, 3354.

[89] George, P.M.; LaVan, D.A.; Burdick, J.A.; Chen, Ch-Y.; Liang, E.; Langer, R. Adv.Mater. 2006, 18, 577.

[90] Abidian, M.R.; Kim, D-H.; Martin, D.C. Adv. Mater 2006, 18, 405.

[91] Teles, F.R.R.; Fonseca, L.P. Mater. Sci. Eng. C, 2008, 28, 8, 1530.

[92] Ateh, D.D.; Navsaria, H.A.; Vadgama, P. J. Royal. Soc. Interface, 2006, 3, 741.

[93] Nambiar S.; Yeov, J.T.V. Biosensors Bioelectronic, 2011, 26,1825.

[94] Drummomd, T.G.; Hill, M.G.; Barton, J.K. Nature Biotechnol. 2003, 21, 1192.

[95] Wei, F.; Lillehoj, P.B.; Ho, C-M. Pediatric Res 2010, 67, 458.

[96] Cagnin, S.; Carabello, M.; Guiduci, C.; Martini, P.; Ross, M.; SntaAnna, M.; Danley, D.; West, T.; Lannfranchi, G. Sensors 2009, 9, 3122.

[97] Lucarelli, F.; Capponucelli, S.; Marrazza, G.; Sangiorgi, L.; Muscini, M. Analyst 2009, 134, 1, 52.

[98] Galandrova , J.; Labuda, J. Chem. Papers 2009, 63, 1.

[99] Kiilerich-Pederse, K.; Paulson, C.R.; Jain, T.; Roziosnik, N. Biosens. Bioelectron. 2011, 28, 1, 386.

[100] Fan, Y.; Chen, X.; Trigg, A.D.; Tung, C-h.; Kong, J.; Gao, Z. J. Am. Chem. Soc. 2007, 129, 5437.

[101] Sheng, Q.; Wang, J.; Zheng, J.; Xu, Z.; Zhang, H. Biosens. Bioelectronics 2010, 25, 2071.

[102] Fan, Y.; Chen, X.; Trigg, A.D.; Tung, C-h.; Kong, J.; Gao, Z. J.Am.Chem.Soc. 2007, 129, 5437.

[103] Xie, H.; Luo, S-C.; Yu, H-h. Small, 2009, 5, 2611.

[104] Hafaid, I.; Chebil, S.; Kori-Youssoufi, H.; Bessueille, F.; Errachid, A.; Sassi, Z.; Ali, Z.; Abdelghani, A.; Jaffrezic-Renault, N. Sens. Actuators B: Chemical 2010, 144, 323.

[105] Tully, E.; Higson, S.P.; O'Kennedy, R. Biosens. Bioelectronics, 2008, 23, 906.

[106] Lee, I.; Luo, X.; Cui, X.T.;Yun, M. Biosens. Bioelectronics 2011, 26, 3297.

[107] Gode, V.K.; Shirale, D.J.; Gaikward, P.D.; Savale, P.A.; Kakde, K.P.; Kharat, H.J.; Shirsat, M.D. React. Func. Polym. 2006, 66, 1420.

[108] Gaikward, P.D.; Shirale, D.J.; Savale, P.A.; Data, K.; Ghosh, P.; Pathan, A.; Rabbani, G.; Shirsat, M.D. Int. J. Electrochem. 2007, 2, 488.

[109] Arshlan, F.; Ustabas, S.; Arshlan, H. Sensors 2011, 11, 8152.

[110] Yu, E.H., Sundmacher, K. Process Safe Environ. Protect. 2007, 85, B5(), 489.

[111] Senel, M. Synth. Metals, 2011, 161, 1861.

[112] Liu, J.; Agarwal, M.; Varahramyan, K. Sens. Actuat. B-Chem. 2008, 135, 195.

[113] Yoon, H.; Ko, S.; Jang, J. J. Phys. Chem.B. 2008, 112, 9992. 
[114] Yehezkel, O.; Yan, Y-M.; Baravik, I.; Tel-Vered, R.; Willner, I. Chem. Eur. J. 2009, 15, 2674.

[115] Lee, K.N.; Lee, Y.; Son, Y. Elecroanalysis 2011, 23, 9, 2125.

[116] Chen, S.; Fu, P.; Yin, B.; Ycuan, C.; Xiang, Y. Biosyst. Eng. 2011, 34, 711.

[117] Lupu, S.; Lakard, B.; Hihn, J-Y.; Deed, J.; Rougeot, P.; Lallemand, S. Thin Solid Films 2011, 519, 7754. 